\title{
Introducing a Pure Weight-Based Concept for Automatic Test Case Generators
}

This paper was downloaded from TechRxiv (https://www.techrxiv.org).

\section{LICENSE}

CC BY-SA 4.0

SUBMISSION DATE / POSTED DATE

08-08-2021 / 03-02-2022

CITATION

Bazzichi, Franco (2021): Introducing a Pure Weight-Based Concept for Automatic Test Case Generators. TechRxiv. Preprint. https://doi.org/10.36227/techrxiv.15131721.v2

DOI

10.36227/techrxiv.15131721.v2 


\title{
Introducing a Pure Weight-Based Concept for Automatic Test Case Generators
}

\author{
Franco Bazzichi
}

\begin{abstract}
A concept for a weight-based test case generator driven by context-free grammars is introduced, where weights are set for each rule and used as unique driver while deciding which rule to use during the derivation process; we demonstrate why static flat weights generally cannot work but, introducing a simple corrective measure, a dynamic weight-based approach is able to produce random sentences while ensuring coverage and convergence; finally, analyzing the grammar from a new perspective, we describe how it is always possible to define constraints for the identification of balanced weights: balanced weights own the interesting characteristic of making it possible to drive the sentence generation, by a pure weight-based automatic test case generator, of extremely random but finite sentences able to stress the system being tested more extensively than the well-known derivation methods, as confirmed by experimental results. An example of the application of balanced weights approach is included, about a simplified context-free grammar for a theoretical programming language, and possible future directions to strengthen the balanced approach are eventually outlined.
\end{abstract}

Index Terms - Test case generators, Test program generation, Test coverage, Random derivation, Weighted context-free grammars, Probabilistic context-free grammars, Weight-based derivation, Random derivation, Neural network

\section{INTRODUCTION}

TEST case generators applied to context-free grammars have been developed to offer an automatic way to obtain syntactically valid sentences to exercise systems during the test phase; typical target systems are parsers, one component of compilers, but many additional use cases can be identified, namely every system where its input domain can be specified by a context-free grammar (like music notation, equation structure): for such target systems, the advantage of an automatic test case generator is given by its effectiveness against a manual process and, therefore, several tools have been successfully developed and applied in the industry for decades.

One of the required characteristics of test case generators is the ability to satisfy the expected coverage criteria; a taxonomy is presented in [1] including the so called production coverage, which means the usage of all the corresponding rules of the underlying context-free grammar (a criteria which is widely addressed by the majority of existing test case generators to date), PLL and PLR.

Main concern while approaching automatic test case generation is represented by the convergence of the deriva-

\footnotetext{
- F. Bazzichi is at Cadorago (Como) - Italy. E-mail: franco.bazzichi@gmail.com.
}

tion process, and methods to ensure it will be completed in a finite time producing the expected results have to be adopted; a milestone in such a direction is represented by the process described in [2] and refined in more recent papers [3], [4]: in addition to convergence, such a process also owns the interesting feature of generating sentences having the minimal length, even if this feature looks nowadays as just an academic one.

Where automatic test case generators differ from each other is the logic designed to select, between all rules expanding the same nonterminal, the rule to be used to substitute it: while such logic is typically designed to address a specific target coverage (examples are the already mentioned methodology introduced in [2] to address the production coverage, as well as the system introduced more recently in [5] to satisfy PLL coverage), within this paper all the presented approaches share the same logic, and this is simply and uniquely based on weights, introduced in Section 3.

Despite the adopted logic is conceptually so simple, a weight-based test case generator is able to hit different coverage metrics: indeed, exploiting its most relevant characteristic (the capacity to produce random but syntactically valid and finite sentences), the system can be customized to compute the progress towards the target coverage at the end of any sentence derivation, and the generative process is stopped when such progress achieves the expected threshold.

This is considerably simpler than to develop a targetoriented generator and, moreover, in this way a weightbased test case generator even offers the possibility to hit complex coverage metrics for which no generative algorithms have been developed so far, by producing test cas- 
es with the higher combinations of primitive syntax elements and paths.

About convergence of the generative process, different approaches are described in this paper for the weightbased concept, culminated in a formal process to identify which mutual conditions the weights must fulfill in order to be safely coupled with a random selection mechanism.

Beginning with a theoretical flat approach, where all productions have the same chance to be selected, we will add a penalty concept to make a weight-based approach a working solution; then, starting from a simple example and thereafter moving to more complex grammars, we will see how to identify static balanced weights through the identification of formal conditions which have to be satisfied: definition of such conditions, making the penalty concept unnecessary, can be obtained by analyzing the embedded structure of productions and corresponding relationships between nonterminals from a new perspective.

The Balanced approach, described in Section 6, represents the final step of the weight-based proposals introduced in this paper, as it is able to indefinitely generate full random but always finite sentences, ensuring coverage and combined with a very simple logic in selecting the rule to be used during the generative cycles.

Although it is not the main purpose of this paper, that being to illustrate how it is always possible to identify static balanced weights for any grammar morphology, Section 7 contains some experimental results which suggest the potential capacity of the proposed concept to represent a valid alternative against the well-known automatic test case generators, by producing sentences able to address a big variety of coverage metrics.

\section{Definitions}

According to [6] a context free grammar $G$ is a Type-2 grammar defined as a 4-tuple $G=\left(T_{G}, N_{G}, P_{G}, S_{G}\right)$, where:

1. $T_{G}$ is a finite set; elements of $T_{G}$ are called the terminals of $G$; within this paper, the typeface used for terminals is bold;

2. $N_{G}$ is a finite set, disjoint from $T_{G}$; elements of $N_{G}$ are called the nonterminals (or symbols) of $G$; within this paper, the typeface used for nonterminals is italic;

3. $P_{G}$ is a finite relation from $N_{G}$ to $\left(T_{G} \cup N_{G}\right)^{*}$; elements of $P_{G}$ are called the productions (or rules) of $G$, and identified as $p_{i}$; a production $p_{i}$ is expressed here in the form $A \rightarrow \alpha$, where $A$ is an element of $N_{G}, \alpha \in\left(T_{G} \cup N_{G}\right)^{*}$ and $i$ is named index of the rule;

4. $S_{G}$ is an element of $N_{G}$ and is known as the start symbol of $G$ : no productions $A \rightarrow \alpha$ exist where $S_{G} \in \alpha$.
We define the function $\mathcal{R}: A \rightarrow\left\{i \mid p_{i}: A \rightarrow \alpha\right\}$; in other terms, $\mathcal{R}(A)$ corresponds to the set containing the indexes of all rules expanding the nonterminal $A$.

Moreover, we also define the function $\delta: A \rightarrow$ $\left\{A_{j} \in N_{G} \mid \exists p_{i}: A \rightarrow \alpha A_{j} \beta\right\} \cup\left\{\mathcal{S}\left(A_{k}\right) \mid A_{k} \in \mathcal{S}(A)\right\}$; in other terms, $\mathcal{S}(A)$ represents the successors of $A$, meaning the set of symbols contained in all the derivation trees which can be generated starting from nonterminal $A$.

Subsequently, we define as recursive a grammar $G$ where $\exists A \in N_{G} \mid A \in \mathcal{S}(A)$.

The relevance of such a definition is that non-recursive grammars generate finite languages, while recursive grammars generate infinite languages, and this has an impact on the application scope of the different weightbased approaches.

About recursion, we can have two different situations: 1) direct recursion; and 2) indirect recursion.

A symbol $A$ is direct recursive if a rule $A \rightarrow \alpha A \beta$ exists; direct recursion is easy to discover by just looking at productions, one at a time.

To detect indirect recursion we need to look at more productions to discover all the successors: an indirect recursion exists when we can identify a cycle starting from a nonterminal and returning to the same, passing through a non-null sequence of additional nonterminals.

The following is an example of grammar having the cycle $A_{0} \Rightarrow A_{1} \Rightarrow A_{2} \Rightarrow A_{0}$ :

$$
\begin{aligned}
& A_{0} \rightarrow \alpha_{0} A_{1} \beta_{0} \\
& A_{1} \rightarrow \alpha_{1} A_{2} \beta_{1} \\
& A_{2} \rightarrow \alpha_{2} A_{0} \beta_{2}
\end{aligned}
$$

A cycle can also contain sub-cycles: in such case we use a notation like $A_{0} \Rightarrow A_{1} \Rightarrow\left(A_{2} \Rightarrow A_{3}\right)^{*} \Rightarrow A_{0}$ to represent a cycle having 0 or more consecutive repetitions of $A_{2} \Rightarrow A_{3}$.

Thereafter, we define the function $\mathcal{C}: A \rightarrow\left\{A_{i} \mid A_{i} \in\right.$ $\left.\mathcal{S}(A) \wedge A \in \mathcal{S}\left(A_{i}\right)\right\}$ as the cluster of $A$ and, finally, we identify a symbol $A_{1} \in \mathcal{C}(A)$ as entry symbol for the cluster of $A$ if a rule $A_{2} \rightarrow \alpha A_{1} \beta$ exists, where $A_{2} \notin \mathcal{C}(A)$.

\section{INTRODUCING WEIGHTS}

In this paper, all approaches apply the same logic to identify which production has to be used at the time when a nonterminal needs to be replaced, and that logic is simply and uniquely based on weights.

A weighted context-free grammar (or WCFG, as described in [7]) can be defined as a 5-tuple $G=\left(T_{G}, N_{G}, P_{G}, S_{G}, W_{G}\right)$, where $W_{G}$ has a finite relation 1:1 with $P_{G}\left(w_{i}\right.$ identifies the weight set for production $p_{i}$ ).

Weights, having values $0 \leq w_{i} \leq 1$, within this paper define the chance of each $p_{i}$ to be selected: in case $p_{i}$ and $p_{j}$ are 
candidate productions to expand the same nonterminal at some time of a derivation, having $w_{i}>w_{j}$ means that $p_{i}$ has a greater chance to be selected than $p_{j}$ (but this does not mean $p_{i}$ will surely be the actual selected one, being the effective choice determined by the random selection mechanism); if $w_{i}=0, p_{i}$ will never be selected.

Moreover, considering all $p_{i}$ expanding the same symbol, within our weight-based approach the sum of corresponding $w_{i}$ must always be 1 , making this equivalent to the definition of probabilistic context-free grammar (PCFG [7], [8], equally expressive as WCFG); using the function $\mathcal{R}$ introduced in Section 2, such constraint can be expressed as $\forall A \in N_{G}: \sum_{i \in \mathcal{R}(A)} w_{i}=1$.

The definition of weights is completed by specifying their type, which can be static or dynamic: 1) static: $W_{G}$ are kept with their initial values during the overall generative cycles; and 2) dynamic: $W_{G}$ could change while progressing with the generative cycle, depending on some conditions.

\subsection{Weight-based Selection Methods}

Different methods can be designed in order to identify which rule to select to expand a symbol during the derivation process for a weighted context-free grammar; common feature of such methods has to be the adherence to the defined weights meaning that, indicating with $u_{i}$ the current number of utilizations of rule $p_{i}$, for each pair or rules $p_{i}$ and $p_{j}$ expanding the same symbol, on a long term we will have $u_{i} / u_{j} \cong w_{i} / w_{j}$.

An algorithm, which implements a repeatable process while still producing sentences according to weights, can be designed to select the rule $p_{i}$ which mostly keeps the ratio of current utilizations as much as similar to the ratio of weights; assuming that all the $n$ rules expanding the same nonterminal are $p_{k}, \ldots, p_{k+n-1}$, a solution is the following, which at the end identifies $p_{i}$ as rule to be applied:

$$
\begin{aligned}
& \max =-1 \\
& \text { for } j=k \text { to } k+n-1 \text { do } \\
& m=w_{j} \cdot\left(\sum_{l=\boldsymbol{k}}^{\boldsymbol{k}+\boldsymbol{n}-\mathbf{1}} u_{l}+1\right)-u_{j} \\
& \text { if } m>\max \text { then } \\
& i=j \\
& \quad \max =m \\
& \text { end if }
\end{aligned}
$$

An alternative implementation of a weight-based selection method can also be designed using a function (e.g. $r n d())$, generating random real numbers $r(0 \leq r<1)$ with uniform distribution; a possible algorithm is the following:

$$
\begin{aligned}
& \quad r=\operatorname{rnd}() \\
& \quad i=k \\
& \text { sum }=w_{i} \\
& \text { while } \text { sum }<r \text { do } \\
& \quad i=i+1 \\
& \quad \text { sum }=\text { sum }+w_{i}
\end{aligned}
$$

\section{end while}

This latter solution combines the adherence with weights as well as with the greatest level of randomness and, due to its capacity to generate sentences walking through all the possible paths of the grammar, it is the preferred one when we wish to address non trivial coverages (e.g. PLR).

As additional note, programming languages usually own a function to set an initial seed in order to exactly reproduce the same sequence of random numbers being generated: so, in case it would be interesting, setting a seed at the beginning of the generative cycles allows implementing a repeatable process even for the second method, thus obtaining the same output each time the weight-based generator is run.

\section{FLAT}

With Flat we mean a weight-based approach where all productions expanding the same nonterminal always have the same chance to be selected; this means that the Flat approach uses static weights, set to $1 / n$ to all $n$ rules expanding the same symbol.

The Flat approach is always effective in case of nonrecursive grammars (so, for finite languages), as in the following example, introduced in [9]:

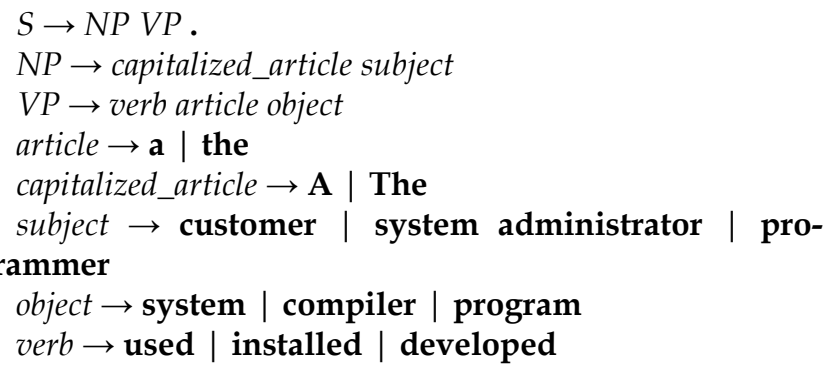

Starting from the nonterminal $S$, rules can be actually selected until no more nonterminals exist; to satisfy the production coverage the generative cycle is repeated from the beginning until all rules are used at least once. Assuming that the random selection mechanism is designed correctly, the overall process always terminates in a finite time.

Moving towards recursive grammars, we have situations where a Flat approach is safely applicable too; let us consider the following grammar, which is recursive due to $p_{3}$ :
1. $S \rightarrow$ id
2. id $\rightarrow$ letter
3. id $\rightarrow$ letter id
4. letter $\rightarrow \mathbf{a}|\mathbf{b}| \ldots \mid \mathbf{z}$

By definition, we have $w_{2}=w_{3}=0.50$ and the process converges as soon as to expand nonterminal $i d$ the production $p_{2}$ is eventually selected instead of $p_{3}$ (and this 
will happen, sooner or later, as $w_{2}>0$ ).

But, in general, the Flat approach does not work as it could lead to a never-ending process; this because convergence during sentence generations is threatened by recursive rules.

As example, we can empirically assess that the Flat approach is not safe for the following recursive grammar $G_{0}$ :
1. $S \rightarrow \exp$
2. $\exp \rightarrow$ term $+\exp$
3. $\exp \rightarrow$ term
4. term $\rightarrow$ id
5. term $\rightarrow(\exp )$

According to the definition, $w_{2}=w_{3}=w_{4}=w_{5}=0.50$; based on that and assuming that the random selection mechanism always identifies $p_{2}$ and $p_{3}$ alternatively (the same for $p_{4}$ and $p_{5}$ ), we see that an endless derivation $S \Rightarrow \exp \Rightarrow$ term $+\exp \Rightarrow \mathbf{i d}+\exp \Rightarrow \mathbf{i d}+$ term $\Rightarrow \mathbf{i d}+(\exp ) \Rightarrow \ldots$ is started, using in sequence rules 1-2-4-3-5: at that point, we are back to the initial situation where nonterminal exp needs to be expanded, meaning that the process is definitely not able to converge.

Section 6 will include a formal proposal to calculate the convergence index, measuring the capacity of a weighted context-free grammar to have finite generation cycles, and we will demonstrate that the Flat approach cannot work for the above grammar.

\section{Flat With Penalties}

A mitigation mechanism to address the weakness of the Flat approach is necessary to ensure the convergence for any context-free grammar using a weight-based approach: with Flat with Penalties we introduce the possibility to dynamically change initial weights by penalties, while generating sentences: the idea is to downgrade weights (i.e. the chance to be selected) of not smart rules as soon as they are used.

For each nonterminal, a rule is identified as smart if it has the shortest derivation length; the process to identify smart rules is very similar to the computation of SLEN as introduced in [2], with the only difference (useful here to address coverage) being for each nonterminal we could have multiple rules identified as smart ${ }^{1}$.

Flat with Penalties uses dynamic weights, initially set by using the same principle of the Flat approach ${ }^{2}$ and, in

1According to the official definition, SLEN is a single-dimension array: always one production only is identified as shortest, even if multiple productions with same length could exist; nevertheless, the additional data structures and the logic adopted to select rules make this irrelevant within [2].

2Choosing adequate initial weights could produce a more balanced output (without deep nesting primarily concentrated at the beginning of case that at the end of a generation cycle not all rules have been used (or used less than the minimum number of wished occurrences), it resumes after all weights have been reset to their initial flat value.

The penalty mechanism works through a configuration parameter $m(0<m<1)$ : every time a non-smart production $p_{i}$ is used, weight $w_{i}$ is reduced by $k=\min \left(w_{i}, m\right)$; at the same time, to respect the weight definition, weight of all $n$ smart rules expanding the same nonterminal is increased by $k / n$; no weights are changed if the selected production is a smart one.

Due to reductions, weight $w_{i}$ for a non-smart rule at some time could be 0 , meaning rule $p_{i}$ will not be selected anymore during the current generation cycle.

No specific criteria are defined to identify the value for the configuration parameter $m$, but it is quite clear that the convergence (end of a generation cycle) is faster for higher values of $m$.

The same mechanism to select a rule for a weighted context-free grammar introduced in Section 3 is used even with Flat with Penalties (all rules act as candidates, smart as well as non-smart), and the unique difference with Flat is because of penalties.

Considering the grammar $G_{0}$ introduced in the previous section, the Flat with Penalties starts setting the same flat weight 0.50 to rules $2 / 3$ and $4 / 5$; about smart, Table I contains the corresponding values for each nonterminal:

\begin{tabular}{ll} 
& $\begin{array}{c}\text { TABLE I } \\
\text { SMART FOR } G_{0}\end{array}$ \\
\hline \hline Nonterminal & Smart \\
\hline$S$ & 1 \\
$\exp$ & 3 \\
term & 4 \\
\hline \hline
\end{tabular}

After that, assuming that the configuration parameter $m$ is set to 0.05 , once the rules 1-2-4-3-5 have been used, the weights will have values as in Table II:$$
\text { TABLE II }
$$

WEIGHTS VALUES FOR $G_{0}$ AFTER 5 STEPS

\begin{tabular}{lll}
\hline \hline Rule & Initial Weight & Current Weight \\
\hline 1 & 1.00 & 1.00 \\
2 & 0.50 & 0.45 \\
3 & 0.50 & 0.55 \\
4 & 0.50 & 0.55 \\
5 & 0.50 & 0.45 \\
\hline \hline
\end{tabular}

In case the random selection mechanism will identify again rules 2 and 5 to expand exp and term (at this stage the difference between $w_{2}$ and $w_{3}$, as well as between $w_{4}$ and $w_{5}$, is not so high), $w_{2}$ and $w_{5}$ will decrease again while $w_{3}$ and $w_{4}$ will rise up correspondingly; so, the

generated sentences), but this option is not addressed in this paper. 
probability of using a smart rule is increased every time a non-smart rule is selected, ensuring the convergence of the generation process.

Hence, just by using one internal data structure (smart) and a very simple process logic, the Flat with Penalties approach is effective and always produces random sentences in a finite time (a higher number of sentences might be required in order to satisfy the production coverage compared with [2]).

\section{BaLANCED}

As it was addressed in Section 4, the Flat approach safely works just for some recursive grammars, as static flat weights (same weight for all rules expanding the same nonterminal) cannot generally ensure the convergence. To resolve such an issue, the Flat with Penalties uses dynamic weights adopting a penalty mechanism.

The Balanced concept represents a step forward, as it defines static weights which always ensure convergence, while eliminating the need to use the penalty mechanism; given any consistent context-free grammar, balanced weights can always be identified and a pure weight-based test case generator can be safely used to obtain full random finite sentences.

The challenge here is how to find such balanced weights: the idea is to analyze the grammar from a new perspective in order to capture the contribution of each rule to change the total number of occurrences of nonterminals in the sentence being generated, and consequently to identify which conditions the weights have to fulfill to be balanced.

\subsection{Delta}

Before outlining how balanced weights can be found, we firstly introduce delta $(\Delta)$, a table where the element $\delta_{i, A}$ contains the resulting difference of occurrences of nonterminal $A$ in the sentence being generated in case $p_{i}: A_{j} \rightarrow \alpha$ would be used; if $A \notin\left\{A_{j}\right\} \cup \alpha, \delta_{i, A}$ is not defined.

Values of $\Delta$ are identified by just observing each rule of $P_{G}$ independently of each other: for rule $p_{i}, \delta_{i, A}$ (whenever is defined) is set to the total number of occurrences of nonterminal $A$ within $\alpha$, reduced by 1 in case $i \in \mathcal{R}(A)$.

As example, referring to the grammar $G_{0}$ introduced in Section 3, Table III shows how $\Delta$ looks like $\left(\delta_{i, A}={ }^{\prime}-\right.$ ' where $\delta_{i, A}$ is not defined):

\begin{tabular}{lccc}
\multicolumn{5}{c}{ TABLE III } \\
\cline { 2 - 3 }$(\Delta)$ FOR $G_{0}$ \\
\hline \hline Rule & $S$ & $\exp$ & term \\
\hline 1 & -1 & +1 & - \\
2 & - & 0 & +1 \\
3 & - & -1 & +1 \\
4 & - & - & -1 \\
5 & - & +1 & -1 \\
\hline \hline
\end{tabular}

Subsequently, using $\Delta$, the contribution of rule $i$ to change the number of total occurrences of symbol $A$ in the sentence being generated can be calculated as $w_{i} \delta_{i, A}$.

\subsection{Constraints, Basics and Advanced}

Next step is the definition of constraints, conditions the weights have to satisfy to represent balanced weights; any set of weights which satisfies all the defined constraints represents a valid set of balanced weights able to ensure that the generation process always ends in a finite time.

A first set of constraints, named basics, descends by the definition of weights, as introduced in Section 3: 1) $\forall A \in N_{G}: \sum_{i \in \mathcal{R}(A)} w_{i}=1$; and 2) $\forall i \in \mathbb{N}|1 \leq i \leq| P_{G} \mid: w_{i}>$ $0^{3}$.

Following that, observing the source grammar from a particular perspective, we also identify advanced constraints which, for each symbol, establish relationships between weights and $\Delta$ : advanced constraints are the most significant components which qualify the Balanced approach.

At this point, let us see how advanced constraints can be defined, starting from a simple grammar $G_{1}$ :
1. $S \rightarrow A$
2. $A \rightarrow \mathbf{a}$
3. $A \rightarrow \mathbf{b} A$
4. $A \rightarrow \mathrm{c} A A$
5. $A \rightarrow \mathrm{d} A A A$

Corresponding values $\Delta_{1}$ are shown in Table IV (column for non-recursive symbols, as by definition the start symbol $S$, can be omitted from $\Delta$ as irrelevant):

\begin{tabular}{lc}
\multicolumn{2}{c}{ TABLE IV } \\
\multicolumn{2}{c}{ DELTA $(\Delta)$ FOR $G_{t}$} \\
\hline \hline Rule & A \\
\hline 1 & +1 \\
2 & -1 \\
3 & 0 \\
4 & +1 \\
5 & +2 \\
\hline \hline
\end{tabular}

Graphically, $G_{1}$ can be represented as in Fig. 1 (characteristics of such representation are described later):

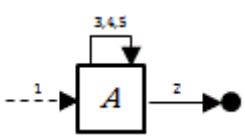

Fig. 1. Graphic representation of $G_{1}$

To define the advanced constraint for symbol $A$, we have to consider how it should be possible to make all of

3In case, for some reasons, we are not interested in coverage or wish to avoid using specific rules, basic constraints of type 2 ) could be $w_{i} \geq 0$ or $W_{i}=0$, respectively; in the paper we always assume $w_{i}>0$. 
its occurrences, as introduced by direct recursive rules, disappear from the generation sentence: this, for grammars not having indirect recursive rules like $G_{1}$, is simply obtained when the overall contribution of rules removing $A$ is higher than the overall contribution of rules adding $A$.

So, in this simple scenario the advanced constraint is represented by condition (1):

$$
\sum_{i \in \mathcal{R}(A)} w_{i} \delta_{i, A}<\mathbf{0}
$$

For simplicity, we identify $\sum_{i \in \mathcal{R}(A)} w_{i} \delta_{i, A}$ as $\sigma_{A}$; therefore, (1) can also be rewritten as follows:

$$
\sigma_{\boldsymbol{A}}<\mathbf{0}
$$

By applying (2) and using the defined $\delta_{i, A}$ as for $\Delta_{1}$, the condition which defines the advanced constraint for $G_{1}$ becomes

$$
\begin{array}{r}
w_{2} \delta_{2, A}+w_{3} \delta_{3, A}+w_{4} \delta_{4, A}+w_{5} \delta_{5, A}<0 \\
\Rightarrow-w_{2}+w_{4}+2 w_{5}<0 .
\end{array}
$$

The fact that $w_{3}$ is not part of the resulting advanced constraint means that productions having $\delta_{i, A}=0$ (i.e. direct recursive rules having just 1 occurrence of expanding symbol on the right side, like $p_{3}$ ) do not influence convergence at all.

Therefore, the entire set of constraints for this grammar is composed by the following conditions:

$$
\left\{\begin{array}{c}
\forall i \in\{1, \ldots, 5\}: w_{i}>0 \\
\sum_{i \in \mathcal{R}(A)} w_{i}=1 \\
-w_{2}+w_{4}+2 w_{5}<0 .
\end{array}\right.
$$

Being the grammar of this example very simple, even the constraints are simple too: this offers the possibility to empirically find valid balanced weights that satisfy all defined constraints, both basics and advanced ones ${ }^{4}$.

Some examples of valid balanced weights are represented by the following sets, for which the corresponding $\sigma_{A}$ are enclosed in brackets: 1) $w_{2}=0.50, w_{3}=0.25, w_{4}=0.10$, $\left.w_{5}=0.15(-0.10) ; 2\right) w_{2}=0.45, w_{3}=0.20, w_{4}=0.30, w_{5}=0.05 \quad(-$ $0.05)$; and 3) $w_{2}=0.45, w_{3}=0.40, w_{4}=0.10, w_{5}=0.05(-0.25)$.

Differences between $\sigma_{A}$ of different sets of balanced weights for symbol $A$ express different speed of convergence, hence the ability to be faster in eliminating all occurrences of $A$; to introduce a measure of such an ability, we define the convergence index $z_{A}$, where higher values mean greater convergence.

4As advanced constraints are disequations, multiple solutions could exist.
Writing (1) as $x<k$, the convergence index expresses the measure of the strength of the disequation, that is how much $x$ is less than $k$; it can be defined by $k-x$ and, in this simple scenario, we consequently have $z_{A}=-\sigma_{A}$.

Convergence is safely ensured where $z>0$; nevertheless, having a higher convergence index may not lead to better results: balanced weights having higher convergence index usually require more generation cycles in order to also satisfy coverage.

Referring uniquely to sets 1),2) and 3), the corresponding convergence indexes $z_{A}$ are, respectively, $0.10,0.05$ and 0.25 . That means with set 3 ) the convergence will be the fastest (generally, as it is depending on the random mechanism), while using set 2) it will be the slowest.

Vice versa, convergence is not ensured, for instance, for set $w_{2}=0.55, w_{3}=0.05, w_{4}=0.10, w_{5}=0.30(0.15)$, set $w_{2}=0.40$, $w_{3}=0.30, w_{4}=0.20, w_{5}=0.10(0)$ and set $w_{2}=0.35, w_{3}=0.10$, $w_{4}=0.30, w_{5}=0.25(0.45)$ because, even if all basic constraints are satisfied, the advanced one is not, meaning they cannot represent valid balanced weights.

They compute $z_{A}$ respectively, as $-0.15,0$ and -0.45 , meaning that, over time, with the first set the occurrences of symbol $A$ in the sentence being generated will increase slightly, while with the last set they will increase greatly; cases where $\sigma_{A}=z_{A}=0$ represent limit situations where the generation cycle never terminates, not being able statistically to definitely remove all the occurrences of symbol $A$ in the generation string.

Nevertheless, due to random mechanism, it could happen that the generation cycle sometimes terminates by using unbalanced weights too, depending on the effective sequence the rules are selected.

Obviously grammars are in general not so simple like $G_{1}$, so now we move a step forward by analyzing $G_{2}$, which includes both direct and indirect recursions.

1. $S \rightarrow E$

2. $E \rightarrow E+T$

3. $E \rightarrow T$

4. $T \rightarrow T^{*} F$

5. $T \rightarrow F$

6. $F \rightarrow$ id

7. $F \rightarrow E$

Corresponding values $\Delta_{2}$ are shown in Table V:

\begin{tabular}{lccc}
\multicolumn{5}{c}{$\begin{array}{c}\text { TABLE V } \\
\text { Delta }(\Delta) \text { FOR } G_{2}\end{array}$} \\
\hline \hline Rule & $E$ & $T$ & $F$ \\
\hline 1 & +1 & - & - \\
2 & 0 & +1 & - \\
3 & -1 & +1 & - \\
4 & - & 0 & +1 \\
5 & - & -1 & +1 \\
6 & - & - & -1 \\
7 & +1 & - & -1 \\
\hline \hline
\end{tabular}


Graphically, we can describe it as in Fig. 2: path from symbol $A_{1}$ to $A_{2}$ exists if a rule $A_{1} \rightarrow \alpha A_{2} \beta$ exists, while paths to black circles represent a rule $A \rightarrow \alpha$ where $\alpha \in T_{G}^{*}$; a graphical representation helps in understanding the logic behind the advanced constraints, as it easily allows to follow the flow from a nonterminal to the next one.

Focusing on symbol $E$ of $G_{2}$, dotted lines represent the path which can reintroduce it (through symbol $F$, when $p_{7}$ is used).

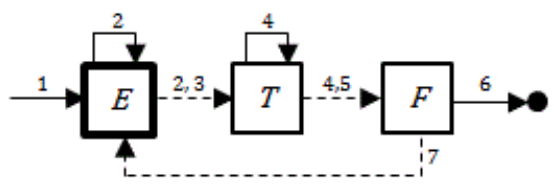

Fig. 2. Graphic representation of $G_{2}$ (focus on $E$ )

In such a similar situation, having the condition (2) as constraint is not enough, as it does not take into account indirect recursive rules either, with the possibility to reintroduce the symbol $E$ : to take care of such indirect contributions, we need to estimate how many symbols $E$ are statistically reintroduced by walking through the recursive path $E \Rightarrow T \Rightarrow F \Rightarrow E$.

To evaluate such indirect contributions, the rally concept is introduced (for symbol $A$, denoted as $\lambda_{A}$ ): the rally specifies the average number of steps which are necessary to statistically exit from expanding an occurrence of a symbol, applying the weight-based selection mechanism.

The rally $\lambda$ is function of $\sigma$, and defined as follows:

$$
\lambda=f(\sigma)=\left\{\begin{aligned}
-\frac{1}{\sigma}, & \sigma<0 \\
+\infty, & \sigma \geq 0 .
\end{aligned}\right.
$$

For $G_{2}$ we have $\lambda_{E}=-1 /\left(w_{2} \delta_{2, E}+w_{3} \delta_{3, E}\right) \Rightarrow \lambda_{E}=1 /$ $w_{3}$; as example, 4 steps are necessary (on average) to exit from expanding $E$ just by using $p_{2}$ and $p_{3}$ in case $w_{3}=0.25$.

The condition defining the advanced constraint for symbol $E$ (part of an indirect recursive path) has to ensure that the capacity to make the symbol $E$ disappear, by applying rules expanding $E$ only, is enough to counteract the impact of the number of occurrences of symbol $E$ introduced for effect of indirect recursive path $E \Rightarrow T \Rightarrow F \Rightarrow E$.

Using the rallies $\lambda_{E}, \lambda_{T}$ and $\lambda_{F}$, the advanced constraint for $E$ of $G_{2}$ can be expressed by

$$
\begin{gathered}
\lambda_{E} \sigma_{E}+\lambda_{E}\left(w_{2} \delta_{2, T}+w_{3} \delta_{3, T}\right) \cdot \lambda_{T}\left(w_{4} \delta_{4, F}+w_{5} \delta_{5, F}\right) \\
\cdot \lambda_{F}\left(w_{7} \delta_{7, E}\right)<0 .
\end{gathered}
$$

The first term of the left side of the constraint, $\lambda_{E} \sigma_{E}$, analyzes the effect of rules expanding $E$, including the direct recursive too.

The remaining terms of the left side, always greater than
0 , analyze the indirect recursive path (defined as cycle) of $G_{2}, E \Rightarrow T \Rightarrow F \Rightarrow E$, and estimate the combined effect of counting:

1. How many occurrences of symbol $T$ are introduced in the string being generated during the $\lambda_{E}$ steps, computed as $\lambda_{E}\left(w_{2} \delta_{2, T}+w_{3} \delta_{3, T}\right)$;

2. For each $T$ introduced by 1 , how many occurrences of symbol $\boldsymbol{F}$ are introduced in the string being generated during the $\lambda_{T}$ steps, computed as $\lambda_{T}\left(w_{4} \delta_{4, F}+\right.$ $\left.w_{5} \delta_{5, F}\right)$;

3. For each $F$ introduced by 2 , how many occurrences of symbol $E$ are reintroduced, due to the $\lambda_{F}$ steps, computed as $\lambda_{F}\left(w_{7} \delta_{7, E}\right)$.

In case an indirect recursive path should not exist, these additional terms do not exist either, and the resulting advanced constraint simplifies into $\lambda_{E} \sigma_{E}<0$, which represents a valid generalization of previous $\sigma_{E}<0$ : indeed, $\lambda_{E} \sigma_{E}$ is less than 0 if and only if $\sigma_{E}<0$ ( $\lambda_{E}$ cannot be negative).

From the definition of rally and assuming $\sigma_{E}<0$ (if not, we have no convergence, even if ignoring the negative effect of indirect recursive rules), the first term is always equal to -1 ; so, the condition for the advanced constraint can be rewritten as follows:

$$
\begin{gathered}
\lambda_{E}\left(w_{2} \delta_{2, T}+w_{3} \delta_{3, T}\right) \cdot \lambda_{T}\left(w_{4} \delta_{4, F}+w_{5} \delta_{5, F}\right) \cdot \lambda_{F}\left(w_{7} \delta_{7, E}\right) \\
<1 .
\end{gathered}
$$

If we wanted now to shift the focus on symbol $T$ of $G_{2}$ (as well as on F), by applying the same logic we would obtain the same condition: indeed, considering a cycle, the advanced constraint is always the same for all symbols which are part of the same cycle, no matter what position they have in the cycle.

The left side of the condition can easily be found by looking at the graphical representation, being the advanced constraint for a cycle simply obtained by the product of elements part of the cycle itself (ignoring direct recursive paths as already embedded in the corresponding $\lambda$ ), where:

1. Name of each nonterminal $A$ is replaced by corresponding $\lambda_{A}$;

2. Indexes over a path ending with nonterminal $A$ are replaced by sum of corresponding $w_{i} \delta_{i, A}$.

In general, considering a cycle without sub-cycles and composed by nonterminals $A_{0}, A_{1}, \ldots, A_{m-1}, A_{m}$ (where $A_{m} \equiv A_{0}$ ), the condition representing the advanced constraint for any of those symbols can be expressed as in (3):

$$
\prod_{k=0}^{m-1} \lambda_{A_{k}} \sum_{i \in \mathcal{R}\left(A_{k}\right)} w_{i} \delta_{k, A_{k+1}}<1 .
$$


According to the definition, in such scenario without sub-cycles the convergence index is calculated as $z=1-$ $\prod_{k=0}^{m-1} \lambda_{A_{k}} \sum_{i \in R\left(A_{k}\right)} w_{i} \delta_{k, A_{k+1}}$.

By applying (3) to $G_{2}$ and using values of $\Delta_{2}$, we obtain the following expressions representing $\lambda_{E}, \lambda_{T}$ and $\lambda_{F}$ : $\lambda_{E}=1 / w_{3}, \lambda_{T}=1 / w_{5}$, and $\lambda_{E}=1$; then, being $\left(w_{2}+\right.$ $\left.w_{3}\right)=\left(w_{4}+w_{5}\right)=1$, the condition representing the advanced constraint for symbols which are part of cycle $E \Rightarrow T$ $\Rightarrow F \Rightarrow E$ becomes

$$
w_{7} /\left(w_{3} w_{5}\right)<1
$$

Therefore, balanced weights for this grammar have to satisfy the following set of constraints (the basics ones and the advanced ones):

$$
\left\{\begin{array}{c}
\forall i \in\{1, \ldots, 7\}: w_{i}>0 \\
\sum_{i \in \mathcal{R}(E)} w_{i}=1 \\
\sum_{i \in \mathcal{R}(T)} w_{i}=1 \\
\sum_{i \in \mathcal{R}(F)} w_{i}=1 \\
w_{7} /\left(w_{3} w_{5}\right)<1 .
\end{array}\right.
$$

The most qualifying condition is represented by the advanced constraints and, due to its simplicity, for $G_{2}$ it is quite easy to empirically find possible solutions, like 1) $w_{2}=0.10, w_{3}=0.90, w_{4}=0.40, w_{5}=0.60, w_{6}=0.47, w_{7}=0.53$ $(z=0.02) ; 2) w_{2}=0.60, w_{3}=0.40, w_{4}=0.70, w_{5}=0.30, w_{6}=0.90$, $w_{7}=0.10 \quad(z=0.17) ;$ and 3) $w_{2}=0.90, w_{3}=0.10, w_{4}=0.10$, $w_{5}=0.90, w_{6}=0.95, w_{7}=0.05(z=0.44)$.

Back to the Flat approach, we are now able to formally demonstrate that it is not safe for $G_{2}$; this can be done just by setting all $w_{i}$ to the flat values, and then to assess if all the constraints are satisfied: by definition, for $G_{2} w_{2}, \ldots, w_{7}$ are all set to 0.50 , which computes $w_{7} /\left(w_{3} w_{5}\right)$ as 2 , formally confirming that the Flat approach does not work for $G_{2}$.

Similarly, about $G_{0}$ introduced in Section 4 , we can now formally assess that the Flat approach is not applicable either: using the same method described above, the resulting qualifying condition is

$$
\lambda_{\exp }\left(w_{2} \delta_{2, \text { term }}+w_{3} \delta_{3, \text { term }}\right) \cdot \lambda_{\text {term }}\left(w_{4} \delta_{4, \text { exp }}\right)<1 .
$$

Such a condition is not satisfied, as the left side is equal to 1: being convergence index $z=0$, representing the limit condition for non-convergence (ensured where $z>0$ ), it formally confirms the previous empirical deduction.

Let us now consider a grammar with multiple clusters, each one containing one or more distinct cycles (e.g.: $A \Rightarrow B$ $\Rightarrow A$ and $A \Rightarrow B \Rightarrow C \Rightarrow A$ for the cluster $\{A, B, C\})$ as in Fig. 3:

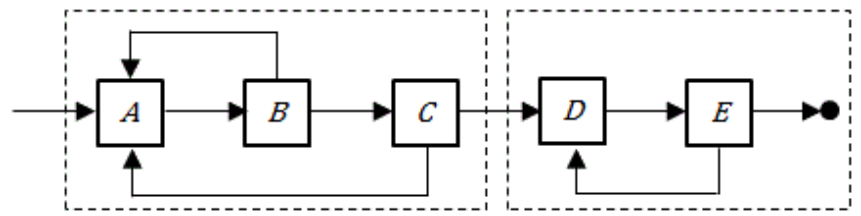

Fig. 3. A grammar with 2 clusters where a cluster has 2 cycles

For each cluster, we will have as many advanced constraints as the distinct entry symbols, where each one is obtained by adding the expressions about the cycles sharing the same entry symbol: for the above grammar we have 2 entry symbols $(A, D)$, and therefore we will have 2 advanced constraints, where the first one for cluster $\{A, B, C\}$ is obtained by adding the expressions about cycles $A \Rightarrow B \Rightarrow$ $A$ and $A \Rightarrow B \Rightarrow C \Rightarrow A$.

To make another up-shift in grammar complexity, let us now see how morphology as in Fig. 4, which includes a sub-cycle, is addressed within the advanced constraint definition.

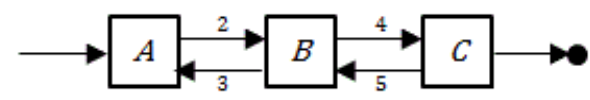

Fig. 4. A grammar with sub-cycle $(C \Rightarrow B)^{\star}$

Denoting with $t$ the result of the known expression $\prod_{k=0}^{m-1} \lambda_{A_{k}} \sum_{i \in R\left(A_{k}\right)} w_{i} \delta_{k, A_{k+1}}$ limited to the $m$ symbols part of the sub-cycle (for the above grammar $t=\lambda_{C} w_{5} \delta_{5, B}$. $\left.\lambda_{4} w_{4} \delta_{4, C}\right)$, the overall contribution considering all the possible repetitions of the sub-cycle will result as $\sum_{i=0}^{\infty} t^{i}$.

Condition (3) needs to be satisfied for the sub-cycle too (if not, we have no convergence). Accordingly, for each sub-cycle an advanced constraint $t<1$ is added.

As the expression $\sum_{i=0}^{\infty} t^{i}$ can be rewritten as $1 /(1-t)$ when $t<1$, every time a sub-cycle is encountered such expression $1 /(1-t)$ replaces correspondent terms in the advanced constraint for the main cycle (in case of nested subcycles, the process is recursively applied).

\subsection{Example}

As a last example, and concrete application of all the above definitions, we introduce now $G_{3}$, a context-free grammar representing a subset of a typical programming language ${ }^{5}$ : we will see how advanced constraints look like, we will identify valid balanced weights and, finally, we will show a sample of generated sentences.

1. $S \rightarrow$ body

2. body $\rightarrow$ begin stm_list end

3. stm_list $\rightarrow$ stm

4. stm_list $\rightarrow$ stm; stm_list 


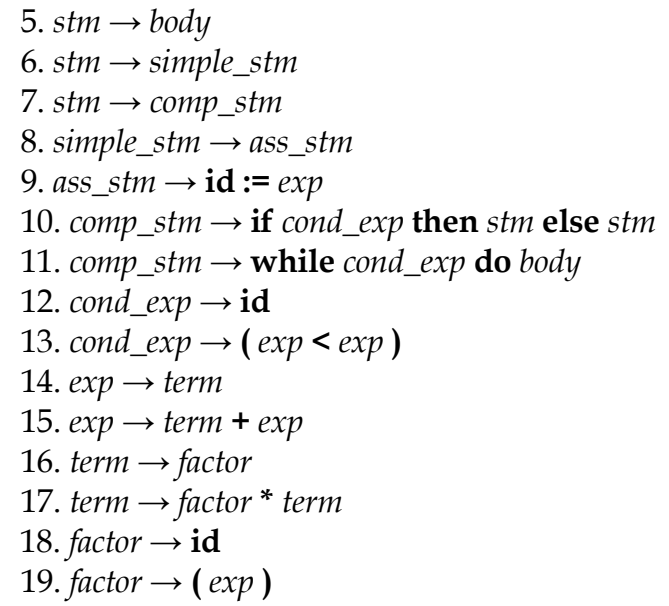

Values of $\Delta$ for this grammar are shown in Table VI (as usual, only recursive symbols have been included as columns, because relevant for the identification of advanced constraints, opposite to non-recursive ones):

\begin{tabular}{lccccccc}
\multicolumn{7}{c}{ TABLE VI } \\
\hline DeLTA $(\Delta)$ FOR G $G_{s}$ \\
\hline \hline Rule & body & stm_list & stm & comp_stm & exp & term & factor \\
\hline 1 & +1 & - & - & - & - & - & - \\
2 & -1 & +1 & - & - & - & - & - \\
3 & - & -1 & +1 & - & - & - & - \\
4 & - & 0 & +1 & - & - & - & - \\
5 & +1 & - & -1 & - & - & - & - \\
6 & - & - & -1 & - & - & - & - \\
7 & - & - & -1 & +1 & - & - & - \\
8 & - & - & - & - & - & - & - \\
9 & - & - & - & - & +1 & - & - \\
10 & - & - & +2 & -1 & - & - & - \\
11 & +1 & - & - & -1 & - & - & - \\
12 & - & - & - & - & - & - & - \\
13 & - & - & - & - & +2 & - & - \\
14 & - & - & - & - & -1 & +1 & - \\
15 & - & - & - & - & 0 & +1 & - \\
16 & - & - & - & - & - & -1 & +1 \\
17 & - & - & - & - & - & 0 & +1 \\
18 & - & - & - & - & - & - & -1 \\
19 & - & - & - & - & +1 & - & -1 \\
\hline \hline
\end{tabular}

The grammar can graphically be represented as in Fig. 5 , where dotted rectangles refer to the 2 embedded distinct clusters: $\gamma_{1}$, composed by symbols \{body, stm_list, stm, comp_stm $\}$, and $\gamma_{2}$, composed by symbols $\{$ exp, term, factor\}:

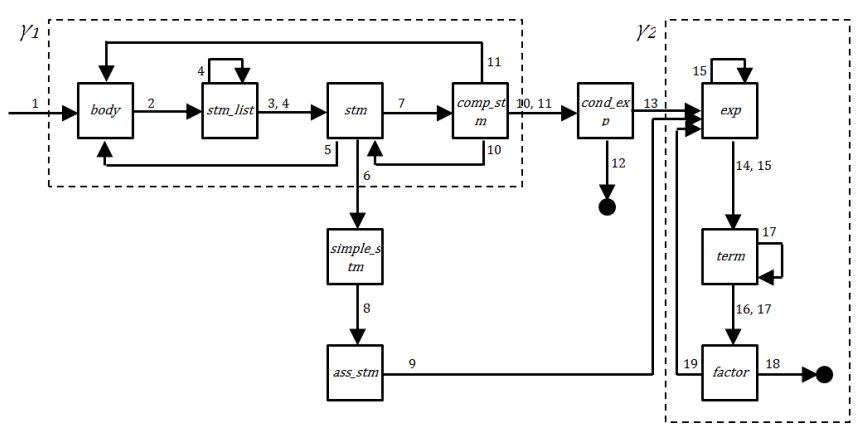

Fig. 5. Graphic representation of $G_{3}$

Cluster $\gamma_{1}$ contains 2 cycles, each one with a sub-cycle, both sharing the same entry symbol (body):

a) body $\Rightarrow s t m \_l i s t \Rightarrow s t m \Rightarrow(\text { comp_stm } \Rightarrow s t m)^{*} \Rightarrow$ body; b) body $\Rightarrow$ stm_list $\Rightarrow$ stm $\Rightarrow$ comp_stm $\Rightarrow$ (stm $\Rightarrow$ comp_stm ${ }^{*} \Rightarrow$ body.

Instead, cluster $\gamma_{2}$ contains 1 cycle only without subcycles: $\exp \Rightarrow$ term $\Rightarrow$ factor $\Rightarrow \exp$.

By applying the construction rules for the morphology of the above cycles / sub-cycles, we obtain the following corresponding conditions, respectively (as the components of sub-cycles in a) and b) of cluster $\gamma_{1}$ are the same, the corresponding constraints will be the same too, and therefore one single condition will be listed below for both):

$$
\begin{aligned}
& \lambda_{b d} w_{2} \delta_{2, s l} \cdot \lambda_{s l}\left(w_{3} \delta_{3, s t}+w_{4} \delta_{4, s t}\right) \\
& \cdot \lambda_{s t} w_{5} \delta_{5, b d} /\left(1-\lambda_{s t} w_{7} \delta_{7, c s} \cdot \lambda_{c s} w_{10} \delta_{10, s t}\right)+\lambda_{b d} w_{2} \delta_{2, s l} \\
& \cdot \lambda_{s l}\left(w_{3} \delta_{3, s t}+w_{4} \delta_{4, s t}\right) \cdot \lambda_{s t} w_{7} \delta_{7, c s} \\
& \cdot \lambda_{c s} w_{11} \delta_{11, b d} /\left(1-\lambda_{c s} w_{10} \delta_{10, s t} \cdot \lambda_{s t} w_{7} \delta_{7, c s}\right) \lambda_{c s} w_{10} \delta_{10, s t} \\
& <1 ; \quad \lambda_{s t} w_{7} \delta_{7, c s} \cdot \lambda_{c s} w_{10} \delta_{10, s t}<1 ; \\
& \lambda_{e x}\left(w_{14} \delta_{14, t e}+w_{15} \delta_{15, t e}\right) \cdot \lambda_{t e}\left(w_{16} \delta_{16 . f c}+w_{17} \delta_{17, f c}\right) \\
& \cdot \lambda_{f c}\left(w_{19} \delta_{19, e x}\right)<1 .
\end{aligned}
$$

Subsequently, using the values of $\Delta$ from Table VI, substituting the rallies with corresponding expressions and, moreover, exploiting the basics constraints definition, advanced constraints for $\gamma_{1}$ and $\gamma_{2}$ can eventually be, respectively, rewritten as:

$$
\begin{gathered}
\left(w_{5}+w_{7} w_{11}\right) /\left[w_{3} \cdot\left(1-2 w_{7} w_{10}\right)\right]<1 \\
2 w_{7} w_{10}<1 \\
w_{19} /\left(w_{14} w_{16}\right)<1 .
\end{gathered}
$$

To identify valid solutions, other than empirically (not so easy when we have such a complex advanced constraints), it is always possible to utilize a byproduct of the Flat with Penalties approach: indeed, at the end of generation cycles, relative percentages of usage of each rule expanding the same symbol always represent safe values to be used as balanced weights.

Anyhow, starting from a valid set of balanced weights, manual changes can be made, for instance to drive the sentence generation to increase or to reduce the nesting level caused by recursive rules: in any case, after a manual modification, the new set needs to be re-validated to assess if all the basics as well as the advanced constraints are still satisfied.

A valid solution is represented by the following set of weights, simply obtained by running the Flat with Penalties approach with parameter $m=0.01$ over $G_{3}: w_{3}=0.65$, $w_{4}=0.35, w_{5}=0.29, w_{6}=0.42, w_{7}=0.29, w_{10}=0.29, w_{11}=0.71$, $w_{12}=0.43, w_{13}=0.57, w_{14}=0.65, w_{15}=0.35, w_{16}=0.66, w_{17}=0.34$, $w_{18}=0.72, w_{19}=0.28$.

The above weights satisfy all the conditions being the 
expressions on the left side representing advanced constraints for clusters $\gamma_{1}$ and $\gamma_{2}$ evaluate, respectively, to 0.92 and 0.65 , both less than 1 (with corresponding convergence index of 0.08 and 0.35 ): this means that they are balanced and we can safely run an automatic pure weight-based test case generator to always obtain finite sentences.

Below, a real set of sentences generated by a pure weight-based test case generator applied to $G_{3}$ (enriched with formatting directives) with the mentioned balanced weights, where we can see its ability in generating extensively random (but always finite) test cases.

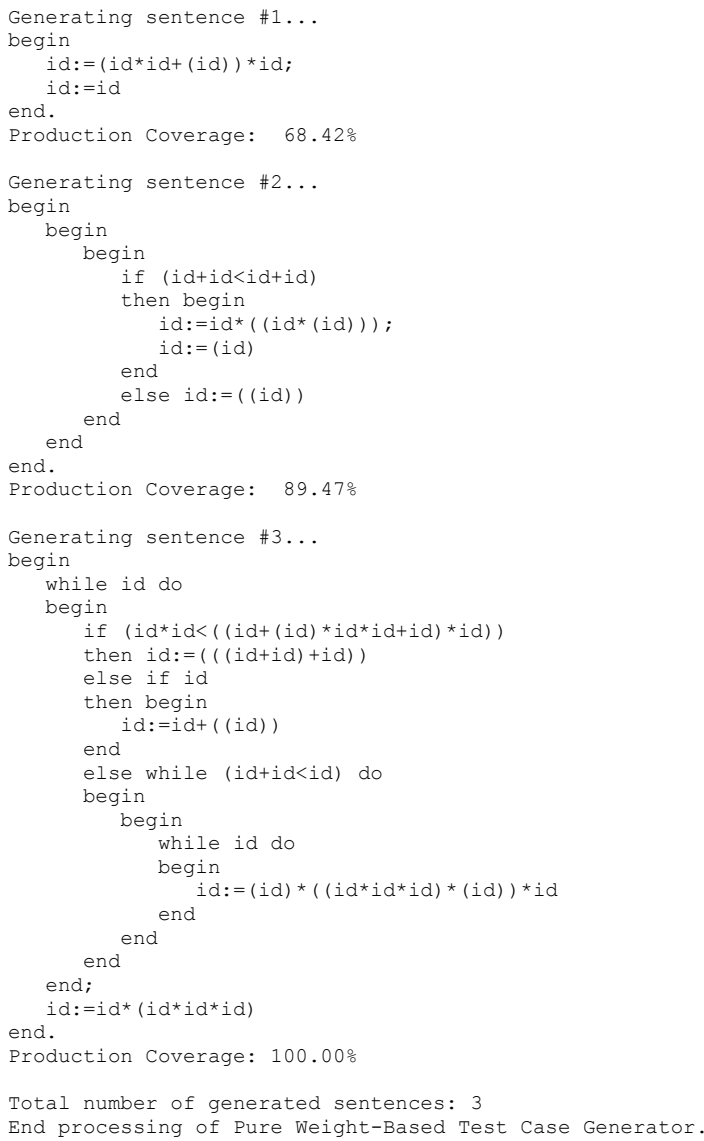

Instead, the following output is produced by the wellknown method [2] (mentioned hereafter as reference meth$o d)$ over the same grammar $G_{3}$ :

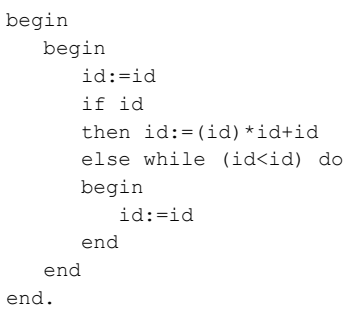

The next section includes some experimental results executed to perform a rough comparison between these two methods as well as to outline how the weight-based generator is able to address a large variety of coverage metrics.

\section{EXPERIMENTAL RESULTS}

The weight-based test case generator has been implemented in Free Pascal IDE 1.0.12 and the main unit, which includes the derivation logic, is composed by less than 100 lines of code. The main unit includes both versions of the selection methods described in Section 3.1 and has been designed to run either the Flat or the Balanced approach; which selection method and approach have to be used during a run are driven by input parameters.

Multiple tests have been conducted on grammars with different level of complexity (e.g. a morphology with 5 nested sub-cycles and multiple entry symbols): the construction of constraints as described in Section 6.2 turned out to be correct as the process always ended in a finite time using any set of weights causing the condition expressing the constraints to be satisfied and, vice versa, the process diverged when it was not satisfied.

By running the weight-based test case generator over $G_{3}$ in order to fulfill the production coverage metric with the weights indicated in Section 6.3, we see that it requires 2.80 sentences $^{6}$ to reach the score of $100 \%$, opposite to 1 as performed by the reference method (on the other hand, this is exactly its primary goal).

By the way, such 2.80 random sentences have been, at the same time, able to better address much stronger coverage metrics like PLR (respectively, $91.57 \%$ vs $69.66 \%$ ). As the reference method always generates the same set of test cases, its coverage score cannot increase by multiple runs; vice versa, the weight-based method can improve the score at each run until a full coverage of $100 \%$ is reached (or any different predefined threshold), obtained for $G_{3}$ with 11.25 sentences.

Moreover, the power of the weight-based generator can be exploited also to address new coverage metrics: just as an example, we define as $P L R+$ a coverage metric which extends $P L R$ as defined in [1], requiring that the test cases also need to exercise all the "reduce" actions of the corresponding LR automata. Indeed, the weightbased generator has been proved able of achieving $100 \%$ of $P L R+$ over $G_{3}$ with 11.55 sentences, reaching the score of $90 \%$ just after 4.75 sentences.

\section{ConClusions}

A demonstration about effective applicability of an automatic test case generator for context-free grammar where the unique criterion to select a rule is based on weights has been provided. The weight-based approaches differentiate against well-known methods as they easily generate sentences in a very random way while not requiring

\footnotetext{
6Data reported here about the weight-based approach (number of sentences, scores) are an average obtained through a significant volume of not repeatable generation cycles.
} 
internal data structures as well as complex logic to run.

Convergence of the generation cycles for weight-based methods can be safely ensured by using a penalty mechanism or using balanced weights: how to identify constraints defining balanced weights according to the typical morphologies a grammar can contain has been presented.

An example of generated sentences produced by using the Balanced method has been provided for a context-free grammar representing a reduced portion for a theoretical programming language; the example provides a clear understanding on how the method is capable to generate large but finite full random sentences, while ensuring coverage and convergence.

This represents a step forward in automatic test case generation as, using a very simple derivation logic, it offers a greater chance to extensively exercise the internal design of system being tested, easily addressing very complex coverage metrics, compared with existing wellknown methods as confirmed by experimental results.

Possible further investigation is the development of an automatic engine to define the advanced constraints in order to eliminate the manual analysis of the grammar structure.

Moreover, although we have seen how the Flat with Penalties approach can be exploited to identify a valid set of balanced weights, another interesting area of investigation could be represented by the usage of neural network models, in order to iterate over the defined constraints to eventually obtain, starting from flat weights, valid balanced solutions (weights satisfying all the constraints): in this respect, we notice an interesting isomorphism between the elements of a neural network, the input, the parameters, the activation function and the loss function with delta, the weights, the constraints and the convergence index.

\section{ACKNOWLEDGMENT}

The author wishes to thank his family for the patience during the writing and his cats, which have always been sitting nearby throughout the period.

\section{REFERENCES}

[1] M.H. Esterhuizen, "Test Case Generation for Context Free Grammars", PhD thesis, Faculty of Science, Stellenbosch University, Mar. 2018

[2] P. Purdom, "A sentence generator for testing parsers," BIT Numerical Mathematics, pp.366-375, 1972

[3] B.A. Malloy and J.F. Power, “An Interpretation of Purdom's Algorithm for Automatic Generation of Test Cases", $1^{\text {st }}$ Annual International Conference on Computer and Information Science, Orlando, FL, 2001

[4] A.M. Paracha and F. Franek, "Testing grammars for top-down parsers", Innovations and Advances in Computer Sciences and Engineering, Springer Netherlands, pp. 451-456, 2010
[5] S.V. Zelenov and S.A. Zelenova, "Generation of Positive and Negative Tests for Parsers", Programming and Computer Software, Vol. 31, No. 6, pp. 310-320, 2005

[6] N. Chomsky, "Three models for the description of language", IRE Transaction on Information Theory, Vol. 2, pp. 113-124, 1956

[7] M. Gabor, W. Wieczorek and O. Unold, "Split-Based Algorithm for Weighted Context-Free Grammar Induction", Applied Sciences, Vol. 11, Issue 3, 2021

[8] Z. Chi, "Statistical properties of probabilistic context-free grammars", Computational Linguistics, Vol. 25, pp. 131-160, 1999

[9] D. Baturin, "BNFGen", https://baturin.org/tools/bnfgen

Franco Bazzichi was born in Forte dei Marmi (Lucca), Italy, on March 24, 1955. He received the degree in Computational Science, University of Pisa, Pisa (Italy), 1980.

From 1980 to 1981, consultant for Olivetti and Italtel-SIT; from 1982 to 1984 software developer at Italtel-SIT, from 1984 to 1997 IT training center coordinator and software engineer at Ciba-Geigy, Italy, from 1997 to 2020 he worked for Novartis Farma SpA, Italy, in different areas of Information Technologies, finishing as Country Service Delivery Manager for IT Service Desk and IT Client Platform. During the time he has always kept great interest in software engineering and, recently, in Artificial Intelligence.

Dr. Bazzichi is author, with I. Spadafora, of the paper "An Automatic Generator for Compiler Testing", IEEE TRANSACTIONS ON SOFTWARE ENGINEERING, Volume: SE-8, Issue: 4, July 1982. 